\title{
Interaction of fast and slow varying electromagnetic waves propagating in paraelectric or ferroelectric material
}

\author{
A. I. Maimistov ${ }^{1,2}$ \\ ${ }^{1}$ National Nuclear Research University, Moscow Engineering Physics Institute, Kashirskoe sh. 31, Moscow, \\ 115409 Russia \\ ${ }^{2}$ Moscow Institute of Physics and Technology, Dolgoprudny, Moscow region, 141700 Russia \\ aimaimistov@gmail.com
}

PACS 41.20.Jb, 42.65.-k, 42.65.Sf, 78.20.Bh

DOI 10.17586/2220-8054-2017-8-3-334-338

Once, a referee asked how one can write an oscillator model for a ferroelectric and the total Maxwell equation for a rapidly varying electric field. From what is known about polarization, it is a slow function of time and coordinates, but the optical wave is a fast function. However, there are examples for the interaction of high frequency and low frequency waves in nonlinear wave theory. This means that similar equations can be written for ferroelectric polarization and electromagnetic waves.

Keywords: Maxwell equation, electromagnetic waves, Duffing oscillator model, ferroelectric.

Received: 19 January 2017

Revised: 28 January 2017

\section{Introduction}

The pulse propagation of electromagnetic radiation in different media having a long-range order is an attractive problem of electrodynamics. There are dielectrics having a permanent polarization in the absence of an external electric field, which are similar to magneto-ordered media. This simple class of media is ferroelectric. Another class is the magnetoelectric, where both permanent polarization and permanent magnetization exist, even though the electromagnetic field is absent. A common feature of these media is their highly nonlinear response to the incident electromagnetic wave. Nonlinear optical phenomena in the ferroelectric have been considered. The self-focusing of the few-cycle pulses in ferroelectric [1], the self-interaction processes [2-8], the soliton generation [9-12], the second harmonic generation [13-15] have been investigated in the last ten-fifteen years.

It is important to note that the order parameter of the ferroelectric (i.e., the polarization) in many cases is due to lattice dynamics. The result is a slow varying of the polarization in the optical frequency scale. Thus, electromagnetic wave propagation gives rise to an interaction of the rapidly and slowly varying waves. The aim of this paper to demonstrate that the Ginzburg-Landau-Devonshir model can be adapted for description of the rapidly and slowly varying electromagnetic waves in a ferroelectric medium.

\section{Base equations}

General equations for the description of electromagnetic waves in a ferroelectric material can be written by using the phenomenological equation:

$$
\tau_{0}^{2} \frac{\partial^{2} \mathbf{P}}{\partial t^{2}}+\gamma^{-1} \frac{\partial \mathbf{P}}{\partial t}=-\frac{\delta \Phi}{\delta \mathbf{P}}
$$

where $\Phi=\Phi[\mathbf{P}, \mathbf{E}]$ is Ginzburg-Landau-Devonshir free energy functional [16-20]:

$$
\Phi=\int d z\left\{\frac{1}{2} A \mathbf{P}^{2}+\frac{1}{4} B \mathbf{P}^{4}+\frac{1}{6} C \mathbf{P}^{6}-g \mathbf{E} \cdot \mathbf{P}\right\} .
$$

This equation must be added to the wave equation for the electric field strength $\mathbf{E}[21]$ :

$$
\nabla \times \nabla \times \mathbf{E}-\frac{\mu}{c^{2}} \frac{\partial^{2} \mathbf{E}}{\partial t^{2}}=-\frac{4 \pi \mu}{c^{2}} \frac{\partial^{2} \mathbf{P}}{\partial t^{2}} .
$$

In the one dimensional case and at $C=0$, these equations can be rewritten as:

$$
\begin{aligned}
c^{2} E_{z z} & -E_{t t}=4 \pi P_{t t}, \\
\tau_{0}^{2} P_{t t}+\gamma^{-1} P_{t} & +A P+B P^{3}=g E .
\end{aligned}
$$

Here, $g=\omega_{p}^{2} / 4 \pi-$ coupling constant 
Let us assume that electromagnetic wave consists of a quasi- harmonic wave and an electromagnetic spike (wave without carry wave). This means the spectra of these wave parts are not enveloped. Furthermore, let the following relations be right:

$$
\begin{aligned}
& E(z, t)=E_{s}(z, t)+E_{f}(z, t), \\
& P(z, t)=P_{s}(z, t)+P_{f}(z, t),
\end{aligned}
$$

for a high frequency (HF) $E_{f}, P_{f}$ and for a low frequency (LF) $E_{s}, P_{s}$ components electric field and polarization, where:

$$
\begin{aligned}
E_{f}(z, t) & =\left(E^{+} e^{i k_{1} z}+E^{-} e^{-i k_{1} z}\right) e^{-i \omega_{1} t}+c . c . \\
P_{f}(z, t) & =\left(P^{+} e^{i k_{1} z}+P^{-} e^{-i k_{1} z}\right) e^{-i \omega_{1} t}+c . c .
\end{aligned}
$$

For the nonlinear terms in (4) one can write:

$$
P^{3}=P_{s}^{3}+3 P_{s}^{2} P_{f}+3 P_{s} P_{f}^{2}+P_{f}^{3} .
$$

The third term in this sum can be represented as:

$$
\begin{aligned}
P_{f}^{2}=\left(P^{+} e^{i k_{1} z}+P^{-} e^{-i k_{1} z}\right)^{2} e^{-i 2 \omega_{1} t}+\left(P^{+*} e^{-i k_{1} z}\right. & \left.+P^{-*} e^{i k_{1} z}\right)^{2} e^{i 2 \omega_{1} t} \\
& +2\left(P^{+} e^{i k_{1} z}+P^{-} e^{-i k_{1} z}\right)\left(P^{+*} e^{-i k_{1} z}+P^{-*} e^{i k_{1} z}\right)
\end{aligned}
$$

Hence, this term describes the second harmonic generation (SHG) and the constant field generation. Some part of this expression takes account in LF part of polarization. The phase mismatch is necessary to get effective SHG, otherwise that can be neglected.

The next term can be represented as:

$$
\begin{aligned}
P_{f}^{3}=\left(P^{+} e^{i k_{1} z}+P^{-} e^{-i k_{1} z}\right)^{3} e^{-i 3 \omega_{1} t}+\left(P^{+*} e^{-i k_{1} z}+P^{-*} e^{i k_{1} z}\right)^{3} e^{i 3 \omega_{1} t} & \\
& +3\left(P^{+} e^{i k_{1} z}+P^{-} e^{-i k_{1} z}\right)^{2}\left(P^{+*} e^{-i k_{1} z}+P^{-*} e^{i k_{1} z}\right) e^{-i \omega_{1} t} \\
& +3\left(P^{+} e^{i k_{1} z}+P^{-} e^{-i k_{1} z}\right)\left(P^{+*} e^{-i k_{1} z}+P^{-*} e^{i k_{1} z}\right)^{2} e^{i \omega_{1} t}
\end{aligned}
$$

Some of these terms are corresponded with third harmonic generation and some of them describe the selfinteraction. The third harmonic generation will be neglected as well.

\section{Reduction of wave equations}

The wave equation (3) can be transformed to Fourier form:

$$
\left(-k^{2}+\frac{\omega^{2}}{c^{2}}\right) E(k, \omega)=-\frac{4 \pi \omega^{2}}{c^{2}} P(k, \omega) .
$$

Due to spectra $E_{s}(k, \omega), E_{f}(k, \omega)$ and $P_{s}(k, \omega), P_{f}(k, \omega)$ not being enveloped, the wave equation can be separated into two parts:

$$
\begin{aligned}
& \left(-k^{2}+\frac{\omega^{2}}{c^{2}}\right) E_{s}(k, \omega)=-\frac{4 \pi \omega^{2}}{c^{2}} P_{s}(k, \omega), \\
& \left(-k^{2}+\frac{\omega^{2}}{c^{2}}\right) E_{f}(k, \omega)=-\frac{4 \pi \omega^{2}}{c^{2}} P_{f}(k, \omega) .
\end{aligned}
$$

In the first equation, the frequencies and wave numbers lie near zero, as all functions $E_{s}(k, \omega)$ and $P_{s}(k, \omega)$ are localized near zero variables. In the second equation, the functions $E_{f}(k, \omega)$ and $P_{f}(k, \omega)$ are localized near $\left(\omega_{1}, k_{1}\right),\left(-\omega_{1}, k_{1}\right),\left(\omega_{1},-k_{1}\right)$ and $\left(-\omega_{1},-k_{1}\right)$. Considering the slowly varying envelopes of electric fields and polarizations near the pair of points $\left(\omega_{1}, k_{1}\right)$ and $\left(\omega_{1},-k_{1}\right)$, one can write the following equation:

$$
\left(-\left(k \pm k_{1}\right)+\frac{\left(\omega_{1}+\omega\right)^{2}}{c^{2}}\right) E^{ \pm}(k, \omega)=-\frac{4 \pi\left(\omega_{1}+\omega\right)^{2}}{c^{2}} P^{ \pm}(k, \omega),
$$

where $\omega \ll \omega_{1}, k \ll k_{1}$. This is due to narrow wave pocket of quasiharmonic waves. Taking $k_{1}=\omega_{1} / c$ in to account, this equation can be approximated as:

or

$$
\left(\mp 2 k k_{1}+\frac{2 \omega_{1} \omega}{c^{2}}\right) E^{ \pm}(k, \omega) \approx-\frac{4 \pi \omega_{1}^{2}}{c^{2}} P^{ \pm}(k, \omega),
$$

$$
\left(\mp k+\frac{\omega}{c}\right) E^{ \pm}(k, \omega) \approx-4 \pi k_{1} P^{ \pm}(k, \omega) .
$$


This relation (the dispersion relation) corresponds to following equation:

$$
\pm \frac{\partial E^{ \pm}}{\partial z}+\frac{1}{c} \frac{\partial E^{ \pm}}{\partial t}=2 i \pi k_{1} P^{ \pm}
$$

This is the base equation for the slowly varying envelopes of the HF electric fields $E^{ \pm}(z, t)$. For LF field $E_{s}(z, t)$ the wave equation is used:

$$
\frac{\partial^{2} E_{s}}{\partial z^{2}}-\frac{1}{c^{2}} \frac{\partial^{2} E_{s}}{\partial t^{2}}=\frac{4 \pi}{c^{2}} \frac{\partial^{2} P_{s}}{\partial t^{2}}
$$

These equations are coupled through polarizations.

\section{Reduction of polarization equations}

In both wave equations (8) and (9) the different polarizations presente. The equation for $P_{s}$ can be written in following form:

$$
\tau_{0}^{2} \frac{\partial^{2} P_{s}}{\partial t^{2}}+\gamma^{-1} \frac{\partial P_{s}}{\partial t}+A P_{s}+B\left[P^{3}\right]_{s}=g E_{s}
$$

where $\left[P^{3}\right]_{s}$ is mark of slowly varying part of $P^{3}$. This part must be extracted from (6) and (7), but there are no low frequency terms among (7). Then, taking into account (6), we can write:

$$
\left[P_{f}^{2}\right]_{s}=2\left(P^{+} P^{+*}+P^{-} P^{-*}\right)=2\left(\left|P^{+}\right|^{2}+\left|P^{-}\right|^{2}\right) .
$$

Thus,

$$
\left[P^{3}\right]_{s}=P_{s}^{3}+2\left(\left|P^{+}\right|^{2}+\left|P^{-}\right|^{2}\right) .
$$

Now, we can present the evolution equation for the LF-part of polarization:

$$
\tau_{0}^{2} \frac{\partial^{2} P_{s}}{\partial t^{2}}+\gamma^{-1} \frac{\partial P_{s}}{\partial t}+A P_{s}+B P_{s}^{3}=g E_{s}-6 B P_{s}\left(\left|P^{+}\right|^{2}+\left|P^{-}\right|^{2}\right) .
$$

It is worth noting that the slowly varying polarization evolves under both external electric field $E_{s}$, and the rapidly varying part of the polarization $P^{ \pm}$.

To find equation for slowly varying envelopes of fast part of the polarization, it is necessary for one to take Fourier transformation of the base equation for polarization (4). This leads to:

$$
\left(-\tau_{0}^{2} \omega^{2}-i \gamma^{-1} \omega+A\right) P(\omega)+B \mathcal{F}\left[P^{3}\right](\omega)=g E(\omega) .
$$

The symbol $\mathcal{F}\left[P^{3}\right](\omega)$ denotes Fourier image of polarization in cube. The relative variables in this equation are localized near the points $\left(\omega_{1}, k_{1}\right)$ and $\left(\omega_{1},-k_{1}\right)$ due to narrow wave pockets $E(\omega)$ and $P(\omega)$. This leads to:

$$
\left(-\tau_{0}^{2}(\omega+\omega)^{2}-i \gamma^{-1}\left(\omega_{1}+\omega\right)+A\right) P^{ \pm}(\omega)+B \mathcal{F}\left[P^{3}\right]^{ \pm}(\omega)=g E^{ \pm}(\omega) .
$$

In the general case (the Fourier transformation is linear one), we get:

$$
\mathcal{F}\left[P^{3}\right]=\mathcal{F}\left[P_{s}^{3}\right]+3 \mathcal{F}\left[P_{s}^{2} P_{f}\right]+3 \mathcal{F}\left[P_{s} P_{f}^{2}\right]+\mathcal{F}\left[P_{f}^{3}\right] .
$$

The first term is not zero only near $\omega=0$, hence this term is not the HF-term. The second term can be written approximately as:

$$
3 \mathcal{F}\left[P_{s}^{2} P_{f}\right] \approx 3 P_{s}^{2} \mathcal{F}\left[P_{f}\right] .
$$

The third term $\mathcal{F}\left[P_{s} P_{f}^{2}\right]$ is approximately $P_{s} \mathcal{F}\left[P_{f}^{2}\right]$. This term is localized near $\omega= \pm \omega_{1}$ and $\omega=0$. Hence, this term is irrelevant. The SHG is not under consideration. The term $\mathcal{F}\left[P_{f}^{3}\right]$ contents the parts localized near $\pm 3 \omega$ and $\pm \omega$. Among the all terms in $P_{f}^{3}$ relevant ones are following

$$
P_{f}^{3}=3\left[\left|P^{+}\right|^{2} P^{+}+2\left|P^{-}\right|^{2} P^{+}\right] e^{-i \omega_{1} t+i k_{1} z}+3\left[\left|P^{-}\right|^{2} P^{-}+2\left|P^{+}\right|^{2} P^{-}\right] e^{-i \omega_{1} t-i k_{1} z}+\text { c.c. }+\ldots,
$$

where dots indicate terms, containing $e^{i \omega_{1} \pm i 3 k_{1} z}$.

Thus, the equation is:

$$
\left[-\tau_{0}^{2}(\omega+\omega)^{2}-i \gamma^{-1}\left(\omega_{1}+\omega\right)+A\right] P^{ \pm}(\omega)+3 B P_{s}^{2} P^{ \pm}(\omega)+3 B\left(\left|P^{ \pm}(\omega)\right|^{2}+2\left|P^{\mp}(\omega)\right|^{2}\right) P^{ \pm}(\omega)=g E^{ \pm}(\omega) .
$$

Taking into account $\omega \ll \omega_{1}$ this equation can be reduced to: $\left(\gamma^{-1}-i 2 \tau_{0}^{2} \omega_{1}\right)(-i \omega) P^{ \pm}(\omega)+\left(A-\tau_{0}^{2} \omega_{1}^{2}-i \gamma \omega_{1}\right) P^{ \pm}(\omega)+3 B P_{s}^{2} P^{ \pm}(\omega)+3 B\left(\left|P^{ \pm}(\omega)\right|^{2}+2\left|P^{\mp}(\omega)\right|^{2}\right) P^{ \pm}(\omega)=g E^{ \pm}(\omega)$.

Inverse Fourier transformation for this expression results in the equation:

$$
\left(\gamma^{-1}-i 2 \tau_{0}^{2} \omega_{1}\right) \frac{\partial P^{ \pm}}{\partial t}+\left(A-\tau_{0}^{2} \omega_{1}^{2}-i \gamma \omega_{1}\right) P^{ \pm}+3 B P_{s}^{2} P^{ \pm}+3 B\left(\left|P^{ \pm}\right|^{2}+2\left|P^{\mp}\right|^{2}\right) P^{ \pm}=g E^{ \pm}
$$


If introduce new parameters: the renormalized relaxation time $\tau_{1}=2 \tau_{0}^{2} \omega_{1}-i \gamma^{-1}$ and the renormalized Landau parameter $A_{1}=A-\tau_{0}^{2} \omega_{1}^{2}-i \omega_{1} \gamma^{-1}$, then the equation for slowly varying part of polarization takes the form:

$$
-i \tau_{1} \frac{\partial P^{ \pm}}{\partial t}+A_{1} P^{ \pm}+3 B P_{s}^{2} P^{ \pm}+3 B\left(\left|P^{ \pm}\right|^{2}+2\left|P^{\mp}\right|^{2}\right) P^{ \pm}=g E^{ \pm} .
$$

The equations (8) and (12) describe the forward $\left(E^{+}, P^{+}\right)$and backward $\left(E^{-}, P^{-}\right)$waves propagation, which are coupled by nonlinear interaction.

\section{Unidirectional approximation}

Above, the total Maxwell equation was used for description of two waves propagating in both directions along the $z$-axis. Frequently, propagation in one direction may be considered. In this case, let us denote $E^{+}=\tilde{E}$, $P^{+}=\tilde{P}$. The system of equations is:

$$
\begin{aligned}
& \frac{\partial E_{s}}{\partial z}+\frac{1}{c} \frac{\partial E_{s}}{\partial t}=\frac{2 \pi}{c} \frac{\partial P_{s}}{\partial t} \\
& \tau_{0}^{2} \frac{\partial^{2} P_{s}}{\partial t^{2}}+\gamma^{-1} \frac{\partial P_{s}}{\partial t}+A P_{s}+B P_{s}^{3}=g E_{s}-6 B P_{s}|\tilde{P}|^{2} \\
& \frac{\partial \tilde{E}}{\partial z}+\frac{1}{c} \frac{\partial \tilde{E}}{\partial t}=2 i \pi k_{1} \tilde{P} \\
& -i \tau_{1} \frac{\partial \tilde{P}}{\partial t}+\left(A_{1}+3 B P_{s}^{2}\right) \tilde{P}+3 B|\tilde{P}|^{2} \tilde{P}=g \tilde{E}
\end{aligned}
$$

The equations (13) and (14) are the generation of the Maxwell-Duffing system. The extra term in right hand side of (14) represents the coupling between HF and LF waves.

It should be noted that if one put $\tilde{P}=0, \tilde{E}=0$ and $\gamma^{-1}=0$, the system of equations (13) and (14) will be reduced to model equations, which are used in $[2,3,8]$.

\section{Conclusion}

In this paper, the simplest model of the electromagnetic wave propagation in a one-dimensional ferroelectric medium is considered. The nonlinear properties of the medium leads to coupling between the high frequency and low frequency components for electric field and polarization. In the general case, a high frequency incident optical wave generates both HF and LF responses in the medium. Thus, the interaction of the rapidly and slowly varying electromagnetic waves will take place. The extremely short electromagnetic pulse or the few-cycle pulses generate both high and low frequency components of the polarization in a ferroelectric medium. This results in HF radiation. If this radiation is not taken into consideration, the Maxwell-Duffing model [1,2,9] can be used for describing the extremely short electromagnetic pulse propagation in a one-dimensional ferroelectric medium.

In the $2 \mathrm{D}$ epitaxial ferroelectric islands and in the thin films, the size effects on domain configuration and polarization play a large role in the electromagnetic response $[25,26]$. These effects can be accounted for in the model under consideration.

Here, the parametric processes, for example second harmonic generation, are not considered. However, this can be done with no problem.

The one-dimensional model is considered. Usually, ferroelectric materials are anisotropic crystals. Taking into account of this anisotropy will result in the cumbersome system of equations. It is of interest to investigate nonlinear wave propagation in the framework of these models. The formation of the spatial structures, e.g. the spatial solitons, the light bullets and needles, is expected.

It has been known that in certain substances the ferroelectricity can be of a purely electronic nature [22-24]. The study of nonlinear wave propagation in such media is very attractive because speed of the electron response can be comparable to electromagnetic field variation.

\section{Acknowledgement}

I am grateful to Dr. J. G. Caputo and Dr. E. V. Kazantseva for enlightening discussions. This investigation was supported by the Russian Foundation for Basic Research (Grant No. 15-02-02764) and by the Russian Science Foundation (Project 14-22-00098). 


\section{References}

[1] Nesterov S.V., Sazonov S.V. On self-focusing and defocusing of few-cycle pulses in hydrogen-containing ferroelectrics. Quantum Electron., 2004, 34 (2), P. 151-155.

[2] Kazantseva E.V., Maimistov A.I. Nonlinear Waves in Para- and Ferroelectrics Described by the LandauKhalatnikov Equation. Opt. Spectr., 2005, 99 (1), P. 91-97.

[3] Caputo J.-G., Kazantseva E.V., Maimistov A.I. Electromagnetically induced switching of ferroelectric thin films. Phys. Rev. B, 2007, 75 (1), 014113 (9 pp.).

[4] Caputo J.G. , Kazantseva E.V., Loukitch V, Maimistov A.I. Cavity with an embedded polarized film: an adapted spectral approach. J. Phys. A, 2009, 42 (16), 165204 (18 pp.).

[5] Caputo J.-G., Maimistov A.I., et al. High frequency polarization switching dynamics of a thin ferroelectric film. Phys. Rev. B, 2010, 82, 094113 (8 pp.).

[6] Ozhenko S.S., Kazantseva E.V., Maimistov A.I. proagation of the ultrashort pulses in segnetoelectroc described by the Duffung model of fifth order. Nanosystems: Physics, Chemistry, Mathematics, 2012, 3 (1), P. 117-124.

[7] Subedi A. Proposal for ultrafast switching of ferroelectrics using midinfrared pulses. Phys. Rev. B, 2015,92 (21), 214303.

[8] Kazantseva E.V., Maimistov A.I. On the passage of an extremely short electromagnetic pulse through a ferroelectric layer embedded in a paraelectric. Opt. Spectr., 2012, 113 (5), P. 550-555.

[9] Sazonov S.V. Electromagnetic vide solitons and brithers in the ferroelectric materials of KDP type. FTT, 1995,37 (6), P. 1612-1622 (in Russian).

[10] Khomeriki R., Chotorlishvili L., Malomed B. A., Berakdar J. Creation and amplification of electromagnon solitons by electric field in nanostructured multiferroics. Phys. Rev. B, 2015, 91, 041408(R).

[11] Bandyopadhyay A.K., Ray P.C., Gopalan V. Solitons and critical breakup fields in lithium niobate type uniaxial ferroelectrics. Europ. Phys. Journal B, 2008, 65 (4), P. 525-531.

[12] Hoogeboom C., Kevrekidis P.G., Saxena A., Bishop A.R. Discrete breathers in a nonlinear polarizability model of ferroelectrics. Phys. Rev. E, 2012, 86 (6), 066601 (8 pp.).

[13] Burgel V., Kleemann W., Bianchi U. Optical second-harmonic generation at interfaces of ferroelectric nanoregions in SrSiO 3 :Ca. Phys. Rev. B, 1996, 53 (9), P. 5222-5230.

[14] Yan Sheng, Qian Kong, et al. Theoretical study of Cherenkov-type second-harmonic generation in periodically poled ferroelectric crystals. J. Opt. Soc. Amer. B, 2012, 29 (3), P. 312-318.

[15] Mishina E.D., Sherstyuk N.E., et al. Nonlinear-optical probing of nanosecond ferroelectric switching. Appl. Phys. Lett., 2003, 83 (12), P. 2402-2404.

[16] Landau L.D., Pitaevskii L.P., Lifshitz E.M. Electrodynamics of Continuous Media. Elsevier Butterworth- Heinemann, Oxford, 2000.

[17] Ginzburg V.L. On a dielectric properties of a segnetoelectrics and barium titanate. JETP, 1945, 15, P. 739-742 (in Russian).

[18] Ginzburg V.L. Theory of segnetoelectric phenomena. Usp. Fiz. Nauk, 1949, 38 (8), P. 490-525 (in Russian).

[19] Devonshire A.F. Theory of barium titanate. I. Philos. Mag., 1949, 40, P. 1040.

[20] Ginzburg V.L. Phase transitions in ferroelectrics: some historical remarks. Phys. Usp., 2001, 44 (10), P. 1037-1043.

[21] Born M., Wolf E. Principles of Optics: Electromagnetic Theory of Propagation, Interference and Diffraction of Light. Seventh (expanded) ed., Cambridge Univ. Press, Cambridge, UK, 2003.

[22] Portengen T., Ostreich Th., Sham L.J. Theory of electronic ferroelectricity. Phys. Rev. B, 1996, 54 (24), P. $17452-17463$.

[23] Batista C.D., Gubernatis J.E., Wei-Guo Yin. Electronic mechanism for the coexistence of ferroelectricity and ferromagnetism. Phys. Rev. $B, 2007,75$ (1), 014423 (5 pp.).

[24] Ishihara S. Electronic Ferroelectricity and Frustration. J. Phys. Soc. Jpn., 2010, 79 (01), 011010 (11 pp.).

[25] Jie Wang, Tong-Yi Zhang. Size effects in epitaxial ferroelectric islands and thin films. Phys. Rev. B, 2006,73 (14), 144107 (11 pp.).

[26] Val'kov A.Yu., Kuzmin V.L., et al. Boundary effect on multiple scattering of elastic waves in a half-space. Nanosystems: Physics, Chemistry, Mathematics, 2015, 6 (4), P. 524-536. 